\title{
Longitudinal changes in task-evoked brain responses in Parkinson's disease patients with and without mild cognitive impairment
}

\section{Urban Ekman ${ }^{1,2,3,4}{ }^{4}$, Johan Eriksson ${ }^{1,4}$, Lars Forsgren ${ }^{3}$, Magdalena E. Domellöf $^{3}$, Eva Elgh ${ }^{5}$, Anders Lundquist ${ }^{4,6}$ and Lars Nyberg ${ }^{1,2,4}$}

1 Department of Integrative Medical Biology, Umeå University, Umeå, Sweden

${ }^{2}$ Department of Radiation Sciences, Diagnostic Radiology, Umeå University, Umeå, Sweden

${ }^{3}$ Department of Pharmacology and Clinical Neuroscience, Umeå University, Umeå, Sweden

${ }^{4}$ Umeå Center for Functional Brain Imaging, Umeå University, Umeå, Sweden

${ }^{5}$ Department of Clinical sciences, Psychiatry, Umeå University, Umeå, Sweden

${ }^{6}$ Department of Statistics, USBE, Umeå University, Umeå, Sweden

\section{Edited by:}

Raymond Scott Turner, Georgetown University, USA

Reviewed by:

Peng Lei, The University of

Melbourne, Australia

Dag Aarsland, Karolinska Institute,

Sweden

*Correspondence:

Urban Ekman, Department of

Integrative Medical Biology,

Umeå University, Biologihuset,

SE-901 87 Umeå, Sweden

e-mail: urban.ekman@.umu.se
Cognitive deficits are common in Parkinson's disease. Previous cross-sectional research has demonstrated a link between cognitive impairments and fronto-striatal dopaminergic dysmodulation. However, longitudinal studies that link disease progression with altered task-evoked brain activity are lacking. Therefore, our objective was to longitudinally evaluate working-memory related brain activity changes in Parkinson's disease patients with and without mild cognitive impairment $(\mathrm{MCl})$. Patients were recruited within a longitudinal cohort study of incident patients with idiopathic parkinsonism. We longitudinally (at baseline examination and at 12-months follow-up) compared 28 patients with Parkinson's disease without $\mathrm{MCl}$ with 11 patients with Parkinson's disease and $\mathrm{MCl}$. Functional MRI blood oxygen level dependent signal was measured during a verbal two-back working-memory task. Patients with $\mathrm{MCl}$ under-recruited bilateral medial prefrontal cortex at both time-points (main effect of group: $p<0.001$, uncorrected). Critically, a significant group-by-time interaction effect ( $p<0.001$, uncorrected) was found in the right fusiform gyrus, indicating that working-memory related activity decreased for patients with Parkinson's disease and $\mathrm{MCl}$ between baseline and follow-up, while patients without $\mathrm{MCl}$ were stable across time-points. The functional connectivity between right fusiform gyrus and bilateral caudate nucleus was stronger for patients without $\mathrm{MCl}$ relative to patients with $\mathrm{MCl}$. Our findings support the view that deficits in working-memory updating are related to persistent fronto-striatal under-recruitments in patients with early phase Parkinson's disease and $\mathrm{MCl}$. The longitudinal evolution of $\mathrm{MCl}$ in Parkinson's disease translates into additional task-evoked posterior cortical changes.

Keywords: Parkinson disease, MCl, longitudinal, working memory, functional MRI (fMRI)

\section{INTRODUCTION}

Cognitive impairment frequently accompanies the characteristic motor deficits in Parkinson's disease (PD), ranging from mild cognitive impairment (MCI) to dementia (Kehagia et al., 2010; Svenningsson et al., 2012). Approximately $20-40 \%$ of patients with PD have MCI at an early phase (Aarsland et al., 2010), and the risk of developing PD dementia (PDD) is markedly increased for patients with MCI compared to patients without MCI (Janvin et al., 2006; Broeders et al., 2013; Pedersen et al., 2013).

Previous cross-sectional research has linked cognitive impairments in PD to both structural and functional brain deficits (Kehagia et al., 2010; Svenningsson et al., 2012), and early-phase alterations are commonly related to working-memory and executive processes (Owen, 2004; Monchi et al., 2007; Marklund et al., 2009). In a previous cross-sectional study, we showed that patients with PD and MCI under-recruited bilateral anterior cingulate cortex (ACC) and right caudate nucleus compared with patients without MCI during working-memory updating (Ekman et al., 2012). Additionally, a recent publication reported findings in accordance with our study (Nagano-Saito et al., 2014). Fronto-striatal hypo-activity has also been demonstrated for patients with $\mathrm{PD}$ and impaired executive functions relative to non-impaired patients (Lewis et al., 2003), and impaired working-memory is associated with nigro-striatal (Rinne et al., 2000; Cools, 2006; Ekman et al., 2012), and meso-cortical (Mattay et al., 2002; Monchi et al., 2007) dopaminergic dysfunction.

Cross-sectional studies have thus provided information on the anatomical and neurochemical bases of cognitive deficits in PD. However, longitudinal cohort studies are fundamental in order to better understand underlying mechanisms (Kehagia et al., 2010; 
Monchi and Stoessl, 2012). Research using longitudinal methodology is generally more sensitive in detecting brain changes, and not as susceptible to cohort differences as cross-sectional methodology (Raz, 2005; Nyberg et al., 2010). However, only a limited number of longitudinal studies have linked brain changes to cognitive decline in PD. Functional changes have been related to evolving glucose metabolism decline within cognitive networks of prefrontal- and parietal cortices in patients with PD (Huang et al., 2007), and tests probing posterior cortical function rather than tests probing fronto-striatal cortical function have been demonstrated to enhance predictions of global cognitive decline (Williams-Gray et al., 2007, 2013). Critically, no longitudinal study has previously evaluated task-evoked brain responses in relation to cognitive impairments in PD. Task-evoked methodology offers a unique possibility to evaluate physiological responses within the operative brain in relation to cognitive deficits. Thus, the aim of this study was to longitudinally (at time for initial PD-diagnoses and at 12-months follow-up) assess changes in working-memory related brain responses using functional MRI in a population-based cohort of patients with PD, with and without MCI. Because patients with PD and MCI have increased risk for PDD (Janvin et al., 2006; Broeders et al., 2013), we expect that a large proportion of the patients with MCI eventually progress to PDD. However, although a large proportion of patients with MCI convert to dementia, some patients never progress or even revert to normal cognition, which implies heterogeneous causes (Richard and Brayne, 2014). To ensure that the patients with PD and MCI did not convert to normal cognition or PDD across time (i.e., to increase the prognostic accuracy of MCI in respect to prodromal PDD), repeated neuropsychological testing was carried out (Pedersen et al., 2013). Global cognitive decline might be related to neural dysfunction with posterior cortical basis rather than fronto-striatal circuits (Huang et al., 2007; WilliamsGray et al., 2007). Therefore, we hypothesized that posterior cortical changes across time might be more pronounced than fronto-striatal changes in patients with $\mathrm{PD}$ and $\mathrm{MCI}$, reflecting progression toward prodromal PDD.

\section{MATERIALS AND METHODS PARTICIPANTS}

The recruitment process was conducted within the "newly diagnosed parkinsonism in Umeå” (NYPUM) project, which is a longitudinal population-based cohort study of incident patients with idiopathic parkinsonism, including PD. All physicians in the Umeå catchment area (about 142000 inhabitants) were continually requested for referral of all patients with suspected parkinsonism to the Department of Neurology at Umeå University, during the inclusion interval January 1, 2004, to April 30, 2009. Patients were included if they fulfilled the UK Parkinson's Disease Society Brain Bank clinical diagnostic criteria for definite PD (UKPDS; Gibb and Lees, 1988) at the latest available re-evaluation (36-60 months after initial diagnosis). The participants performed the first (baseline) neuropsychological examination and fMRI scanning approximately 1-2 months after initial PD-diagnosis, and all participants were drug-naïve regarding dopaminergic medication at that time-point (see Table 1 for demographic data). However, at the time for the first follow-up (Mean = 12 months and 23 days, $S D=2$ months and 15 days) all participants (except

Table 1 | Demographics and clinical characteristics.

\begin{tabular}{|c|c|c|c|c|c|c|c|c|}
\hline & \multicolumn{2}{|c|}{$\mathrm{PDMCI}^{+}(n=11)$} & \multicolumn{2}{|c|}{$\mathrm{PDMCI}^{-}(n=28)$} & $\begin{array}{l}\text { Difference } \\
\text { at baseline }\end{array}$ & $\begin{array}{c}\text { Difference at } \\
\text { 12-months follow-up }\end{array}$ & $\begin{array}{l}\text { Main effect } \\
\text { of group }\end{array}$ & $\begin{array}{l}\text { Group-by-time } \\
\text { interaction }\end{array}$ \\
\hline $\begin{array}{l}\text { Male/female (bimanual } \\
p \text {-value) }\end{array}$ & \multicolumn{2}{|c|}{$9 / 2(p=0.07)$} & \multicolumn{2}{|c|}{$13 / 15(p=0.85)$} & n.a. & n.a. & n.a. & n.a. \\
\hline Years of education & \multicolumn{2}{|c|}{9.55 (3.9) } & \multicolumn{2}{|c|}{$12.5(5.4)$} & 0.10 & n.a. & n.a. & n.a. \\
\hline $\begin{array}{l}\text { Age at baseline } \\
\text { examination }\end{array}$ & \multicolumn{2}{|c|}{$65.9(9.0)$} & \multicolumn{2}{|c|}{$67.5(10.2)$} & 0.66 & n.a. & n.a. & n.a. \\
\hline $\begin{array}{l}\text { Years between symptom } \\
\text { onset and PD-diagnosis }\end{array}$ & \multicolumn{2}{|c|}{$2.22(2.2)$} & \multicolumn{2}{|c|}{$2.0(1.5)$} & 0.76 & n.a. & n.a. & n.a. \\
\hline Time-points & Baseline & 12-months & Baseline & 12-months & & & & \\
\hline $\begin{array}{l}\text { Mine-mental state } \\
\text { examination }\end{array}$ & $28.8(1.3)$ & $28.7(1.6)$ & $29.4(0.8)$ & $29.4(0.9)$ & 0.12 & 0.11 & 0.47 & 0.77 \\
\hline UPDRS-III motor scores & $31.5(12.0)$ & $23.2(12.2)$ & $22.9(9.2)$ & $20.3(9.2)$ & 0.01 & 0.21 & 0.91 & 0.47 \\
\hline LED & 0 & $422.7(84.0)$ & 0 & $296.4(159.7)$ & n.a. & 0.01 & n.a. & n.a. \\
\hline MADRS & $4.3(3.7)$ & $2.9(2.1)$ & $4.4(3.4)$ & $3.6(3.7)$ & 0.41 & 0.26 & 0.71 & 0.69 \\
\hline Executive functions & $41.9(5.9)$ & $42.2(15.1)$ & $54.1(10.5)$ & $59.7(14.1)$ & $<0.01$ & $<0.01$ & $<0.01$ & 0.13 \\
\hline Episodic memory & $37.5(8.4)$ & $37.7(7.2)$ & $514(5.3)$ & $52.2(8.3)$ & $<0.01$ & $<0.01$ & $<0.01$ & 0.95 \\
\hline Attention/working memory & $33.9(8.1)$ & $36.2(7.0)$ & $47.4(5.5)$ & $47.3(5,1)$ & $<0.01$ & $<0.01$ & $<0.01$ & 0.21 \\
\hline Language & $51.6(12.5)$ & No values & $52.3(13.4)$ & No values & 0.44 & n.a. & n.a. & n.a. \\
\hline Visuospatial functions & $50.1(9.2)$ & $52.2(4.6)$ & $57.4(3.4)$ & $56.6(5.2)$ & $<0.01$ & 0.02 & $<0.01$ & 0.14 \\
\hline
\end{tabular}

The data are mean values with standard deviations in parentheses. The five cognitive domains for assessing MCl (executive functions, episodic memory, attention/working-memory, language and visuospatial functions) represent means of the included domain-specific tests age-matched $t$-values. $n$, numbers of participants. UPDRS-III (unified Parkinson's disease rating scale, part 3) and LED (levodopa equivalent doses). MADRS (Montgomery and Åsberg Depression Rating scale). n.a., not applicable. Analyses of group differences at baseline, 12-months, main effect of group, and group-by-time interaction are reported as p-values. 
one cognitively healthy participant) received dopaminergic antiparkinsonian medication on a daily basis. Dopamine treatment were recorded and calculated as levodopa equivalent dose (LED) according to the conversion factors used by Tomlinson et al. (2010). This study was approved by the ethics committee of the Faculty of Medicine at Umeå University, Umeå, Sweden.

\section{CLASSIFICATION OF MCI AND FINAL GROUPS}

We classified MCI by assessing The Movement Disorders Society commissioned taskforce criteria for MCI in PD (Litvan et al., 2012) in the same fashion as previously described (Ekman et al., 2012). In brief, the included neuropsychological tests tapped five different cognitive domains: executive functions [Wisconsin Card Sorting Test (WCST) (total errors and perseverative responses) and animal fluency], attention/working-memory (digit span backwards and trail making test, part B), episodic memory [Brief Visuospatial Memory Test revised, (average scores of free and delayed recall); Free and Cued Selective Reminding Test, free recall], visuospatial function (Judgment of Line Orientation Test), and language [Boston Naming Test (BNT)]. Patients who scored $\geq 1.5 S D$ s below the normative age-matched mean value in at least two cognitive test measures were classified with MCI. Because only one cognitive measure was assessed in the language and visuospatial domains, MCI was classified on level I criteria (i.e., subtyping not possible). Because we had available neuropsychological data at 36-monts follow-up, we controlled that results at 36-months follow-up confirmed the MCI classifications set at 12-months to increase accuracy. In conformity with a recent large-scale pooled study (Aarsland et al., 2010), no subjective measures were part of the MCI classification. However, all but one participant classified with MCI reported complaints of subjective cognitive decline. The exclusion process is described in Figure 1. Dementia was assessed using consensus criteria (Emre et al., 2007), reflecting both subjective (deficits severe enough to significantly impair daily life independent of impairment due to non-cognitive symptoms of PD assessed with a clinical interview and the Parkinson's disease questionnaire 39) and objective measures (cognitive impairment in at least two cognitive domains with performance $\geq 2$ SDs below normative age-matched $t$-values; one patient was excluded with suspected dementia of Lewy-Body type). The cut-off for major depression was set to a score of $>17$ (according to the Montgomery and Åsberg Depression Rating scale; Montgomery and Åsberg, 1979), but no participants were excluded due to depression. Patients classified with MCI at baseline but not at 12-, or 36-months follow-up were excluded due to unclear cognitive status. After the initial exclusions, the sample consisted of 39 participants whom all conducted the neuropsychological- and fMRI examinations at both time-points with satisfactory data collection (one participant lacked neuropsychological data at 12-months, but was confirmed as MCI at 36-months). The final groups consisted of 11 participants that had $\mathrm{PD}$ with $\mathrm{MCI}\left(\mathrm{PDMCI}^{+}\right)$at both timepoints, and 28 participants who had $\mathrm{PD}$ without $\mathrm{MCI}\left(\mathrm{PDMCI}^{-}\right)$ at both time-points. Out of the $11 \mathrm{PDMCI}^{+}$participants, seven were treated with levodopa at 12-months follow-up, three with a combination of levodopa and dopamine agonists, and the last

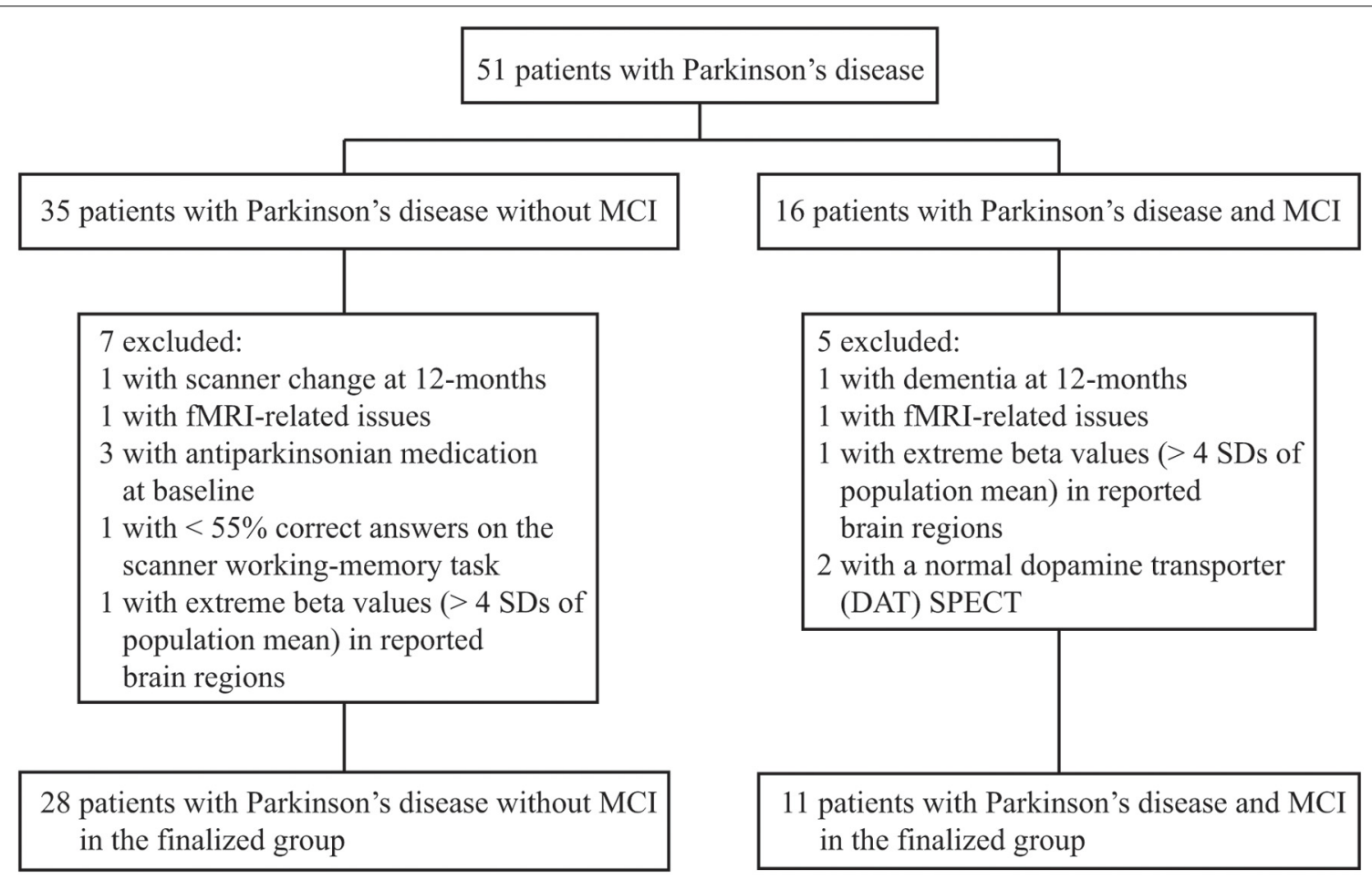

FIGURE 1 | Flow-chart of study profile. Of 67 enrolled Parkinson's disease patients, 51 had conducted neuropsychological assessments and fMRI at both time-points with either a manifested $\mathrm{MCl}$ or were cognitive stable. SD, standard deviation. 
patient with dopamine agonists singly. The equivalent number for $\mathrm{PDMCI}^{-}$were: 12 were treated with dopamine agonists, 10 with levodopa, five with a combination of levodopa and dopamine agonists, and the last patient did not receive any dopaminergic medication at 12-months follow-up.

\section{EXPERIMENTAL PROCEDURES AND IMRI DATA ACOUISITION}

The procedures for the fMRI data acquisition have been described previously (Ekman et al., 2012). In brief, during scanning participants performed a verbal working-memory 2-back updating task. The task demanded the participants to actively maintain and update information (nouns) that regularly were presented on the screen. The participants were instructed to respond "yes" (right index finger) when the presented noun matched the one two items earlier and "no" (left index finger) when it differed, by pushing MRI-compatible keypads. During the baseline condition participants were instructed to do nothing except keeping their gaze fixed on a small circle that were displayed at the center of the screen. The fMRI acquisition was conducted using two different scanners: a 1.5T Philips Intera scanner and a 3T Philips Achieva scanner (both scanners from Philips, Eindhoven, Netherlands). The sequences had the following parameters for the $1.5 \mathrm{~T}$ scanner: repetition time (TR): $3000 \mathrm{~ms}$, echo time (TE) $50 \mathrm{~ms}$, flip angle $90^{\circ}$, field of view: $22 \times 22 \mathrm{~cm}, 64 \times 64$ matrix and $4.40 \mathrm{~mm}$ slice thickness (voxel size $3.44 \times 3.44 \times 4.40 \mathrm{~mm}$ ). During every repetition time, 33 slices were acquired. To avoid effects of signal saturations, five dummy scans were performed prior to the image acquisition. Acquisition parameters for the $3 \mathrm{~T}$ scanner were: TR $1500 \mathrm{~ms}$, TE $30 \mathrm{~ms}$, flip angle $70^{\circ}$, field of view $22 \times 22,64 \times 64$ matrix and $4.65 \mathrm{~mm}$ slice thickness (voxel size $3.44 \times 3.44 \times 4.65)$. During every repetition time, 31 slices were acquired. Ten dummy scans were performed prior to the image acquisition. For $\mathrm{PDMCI}^{-}$the scanner distributions were: 19 participants performed the working memory task in the $1.5 \mathrm{~T}$ scanner and 9 participants in the 3T scanner. The equivalent number for $\mathrm{PDMCI}^{+}$were 6 in the $1.5 \mathrm{~T}$ scanner and 5 in the $3 \mathrm{~T}$ scanner. All included participants conducted the experiment on the same scanner at both time-points.

\section{STATISTICAL ANALYSES}

Preprocessing and statistical analyses of fMRI-data were done with Statistical Parametric Mapping version 8 (SPM 8, Welcome Department of Imaging Neuroscience, London, UK) run in Matlab (MathWorks). The preprocessing included slice-timing correction within each volume to adjust for differences in slice acquisition, Movement correction were performed by realign and unwarp to the first image in the series. To consider groupspecific anatomical brain differences, all participants were initially normalized to the Montreal Neurological Institute (MNI) echoplanar-imaging template. In a second step, normalization was done to group-specific means for patients with and without MCI at each time point. The mean-normalized brain templates were then projected to the total mean of all participants. Finally, the images were smoothed by using an $8 \mathrm{~mm}$ FWHM isotropic Gaussian filter kernel. For functional MRI data, effects were modeled in the framework of the general linear model (GLM; Friston et al., 1995). The event-related fMRI responses were modeled as regressors containing delta functions that represented onsets of word stimuli, and the regressors were convolved with a canonical haemodynamic response function. Model estimations from each participant were input into a second-level factorial analysis with the factors group, time-point, subject, and scanner. Motor scores (UPDRS-III) and levodopa equivalent doses (LED; daily levodopa and dopamine agonist dosages combined) for each participant were input as covariate in the model due to significant group differences (Table 1). Due to the low number of patients treated with dopamine agonists in $\mathrm{PDMCI}^{+}$, no sub-analyses of potential medication effects on cognition were conducted. The statistical threshold was set to $P<0.001$, uncorrected, and the cut-off for number of contiguous voxels $(k)$ was $\geq 10$ in the whole-brain fMRI analyses. Results significant at $p<0.005$ ( $\geq 10$ contiguous voxels) are reported as a trend.

To evaluate possible confounding effects from scanner differences, we performed $F$-tests $(P<0.001)$ on main effect of scanner, group-by-scanner, time-by-scanner, and group-by-timeby-scanner interactions (Stonnington et al., 2008). Pooled analyses have previously been conducted to handle MRI-scanners with different magnetic field strengths (Abdulkadir et al., 2011; Ekman et al., 2012; Marchewka et al., 2014). Furthermore, we analyzed effect sizes (Cohen's $d$ ) on each scanner separately in our reported main effects to ensure equivalence across scanners. One regionof-interest (ROI) analysis in the right caudate was conducted, and the ROI has been previously defined (Ekman et al., 2012).

A psychophysiological interaction (PPI) analysis was performed to evaluate how a ROI functionally interacted with other brain areas during task-performances (Friston et al., 1997). In brief, we performed a second GLM analysis that included one regressor representing the BOLD signal time course in a given ROI, one regressor representing the psychological variable of interest (i.e., working memory condition $>$ baseline condition), and finally one regressor representing the interaction of the former two. The ROIs signal time course (contrast vectors: task condition $>$ baseline condition) for each individual was extracted from a sphere (radius of $6 \mathrm{~mm}$ ) centered on the seeds peak voxel (derived from the group-by-time interaction post-hoc analysis). The statistical threshold in the PPI-analysis was set to $p<0.001$, and the cut-off for number of contiguous voxels was $\geq 10$.

Longitudinal analyses of the right caudate ROI, demographics, clinical characteristics, and the scanner-task behavioral data were assessed within the framework of the GLM using a twofactor (group-by-time) repeated-measures ANOVA. Analyses of group differences at a specific time-point were conducted using independent-samples $t$-test. The statistical threshold in the ROIand the behavioral analyses was set to $p<0.05$. Group comparisons of behavioral performances were analyzed as one-tailed due to á priori hypotheses, and demographic data were analyzed as two-tailed. Gender distribution was analyzed with a binominal test.

\section{RESULTS \\ BEHAVIORAL DATA}

Neuropsychological testing revealed that, $\mathrm{PDMCI}^{+}$had significantly reduced performances (main effect of group across baseline and 12-months) within all cognitive domains (except the 
language domain that was assessed only at baseline) compared to $\mathrm{PDMCI}^{-}$(Table 1). There were no significant group-by-time interaction effects or main effects of time.

As shown in Figure 2, a significant main effect of group was also evident when comparing accuracy (percentage correct answers) on the scanner working-memory 2-back task $\left[F_{(1,36)}=\right.$ 7.12, $p=0.01]$. There was no main effect of group regarding reaction times $\left[\mathrm{RT} ; F_{(1,36)}=0.31, p=0.58\right]$. The group-by-time interaction effect was not significant for performance accuracy $\left[F_{(1,36)}=0.21, p=0.65\right]$, but approached significance for RTs $\left[F_{(1,36)}=3.43, p=0.07\right]$. There was no main effect of time $(p>0.10)$.

\section{NEUROIMAGING DATA \\ Whole-brain analyses}

When comparing working-memory related BOLD-signal intensity, $\mathrm{PDMCI}^{+}$displayed significant under-recruitment compared to $\mathrm{PDMCI}^{-}$. This was seen as a main effect of group in bilateral medial prefrontal cortex, left precentral gyrus, and as a trend in left orbitofrontal cortex, left superior frontal gyrus, right postcentral gyrus, and in the right inferior temporal cortex (Table 2, Figure 3). No overlap was observed between our reported main effects of group and a main effect of scanner analysis, or a group-by-scanner analysis. Furthermore, quantifications of the responses for the two scanners separately showed consistent magnitude of BOLD-signal change on both scanners, with large effect sizes (Cohen's $d>0.80$ ). Less conclusive evidence due to potential influences of the scanner factor was observed in right putamen, left parietal cortex, left cuneus, precuneus, and the left occipital cortex (Supplementary Table 1).

A group-by-time interaction effect was evident in the right fusiform gyrus (Figure 4), right vermis, and as a trend in left inferior temporal cortex (Table 3). Specifically, $\mathrm{PDMCI}^{+}$decreased their BOLD-signal intensity in the fusiform gyrus between baseline and 12-months, while $\mathrm{PDMCI}^{-}$were stable across both time-points. By contrast, $\mathrm{PDMCI}^{+}$increased their BOLD-signal intensity between baseline and 12-months in right parietal cortex and left prefrontal cortex while $\mathrm{PDMCI}^{-}$were again stable across time-points. No overlap was observed between our reported group-by-time interaction effects, and a scanner-by-time interaction, a group-by-scanner interaction, or a group-by-time-byscanner interaction effect. Less conclusive evidence due to potential influences of the scanner factor, was observed in right cerebellum and in left dorsolateral prefrontal cortex (Supplementary Note 1).

We performed a PPI-analysis to evaluate if the right fusiform gyrus had functional connectivity with additional workingmemory related brain regions. A main effect of group revealed that $\mathrm{PDMCI}^{-}$had stronger functional connectivity relative to

Table 2 | Main effects of group in BOLD-signal intensity between patients with Parkinson's disease with and without MCI.

\begin{tabular}{|c|c|c|c|c|}
\hline Brain region & Side & Peak $(x, y, z)$ & $F$ & $\boldsymbol{K}$ \\
\hline \multicolumn{5}{|c|}{ PATIENTS WITHOUT MCI > PATIENTS WITH MCI } \\
\hline \multicolumn{5}{|c|}{ Frontal } \\
\hline Precentral gyrus & $\mathrm{L}$ & $-42,4,30$ & 19.29 & 23 \\
\hline Medial prefrontal & $L / R$ & $0,54,28$ & 22.12 & 148 \\
\hline Orbitofrontal cortex* & L & $-24,34,-12$ & 15.92 & 42 \\
\hline Superior frontal gyrus* & $\mathrm{L}$ & $-22,16,64$ & 14.93 & 24 \\
\hline \multicolumn{5}{|l|}{ Parietal } \\
\hline Postcentral gyrus* & $\mathrm{R}$ & $44,-18,48$ & 14.87 & 48 \\
\hline \multicolumn{5}{|l|}{ Temporal } \\
\hline Inferior temporal* & $\mathrm{R}$ & $48,-18,-22$ & 14.42 & 39 \\
\hline \multicolumn{5}{|c|}{ PATIENTS WITHOUT MCI < PATIENTS WITH MCI } \\
\hline \multicolumn{5}{|c|}{ Frontal } \\
\hline Orbitofrontal cortex & $\mathrm{L}$ & $-8,60,-8$ & 17.28 & 13 \\
\hline \multicolumn{5}{|l|}{ Parietal } \\
\hline Supramarginal & L & $-64,-24,30$ & 17.22 & 13 \\
\hline
\end{tabular}

The coordinates $x, y, z$ refers to the anatomical location of the Montreal Neurological Institute space for the clusters local maxima. $p<0.001$; ${ }^{*} p<0.005$. $L$, left; $R$, right; $F$, F-values; $k$, number of voxels.

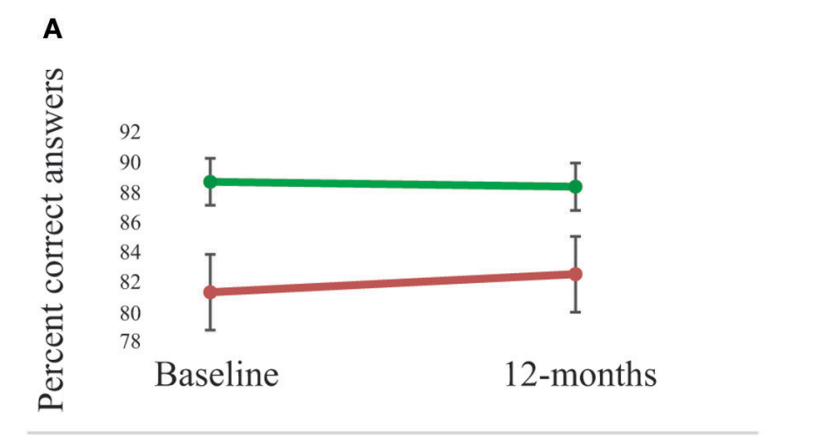

Patients with Parkinson's disease without MCI

FIGURE 2 | Performances on the scanner working-memory task. (A) A significant $(p=0.01)$ main effect of group in performance accuracy was shown with superior performances for patients with Parkinson's disease without $\mathrm{MCl}$ compared to patients with $\mathrm{MCl}$. The
B

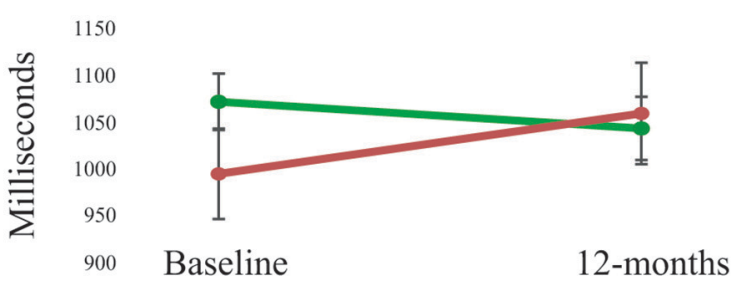

group-by-time interaction effect was not significant $(p=0.65)$. (B) There was no significant group difference in reaction time (main effect, $p=0.58$ ), but the group-by-time interaction effect was close to significant $(p=0.07)$ 

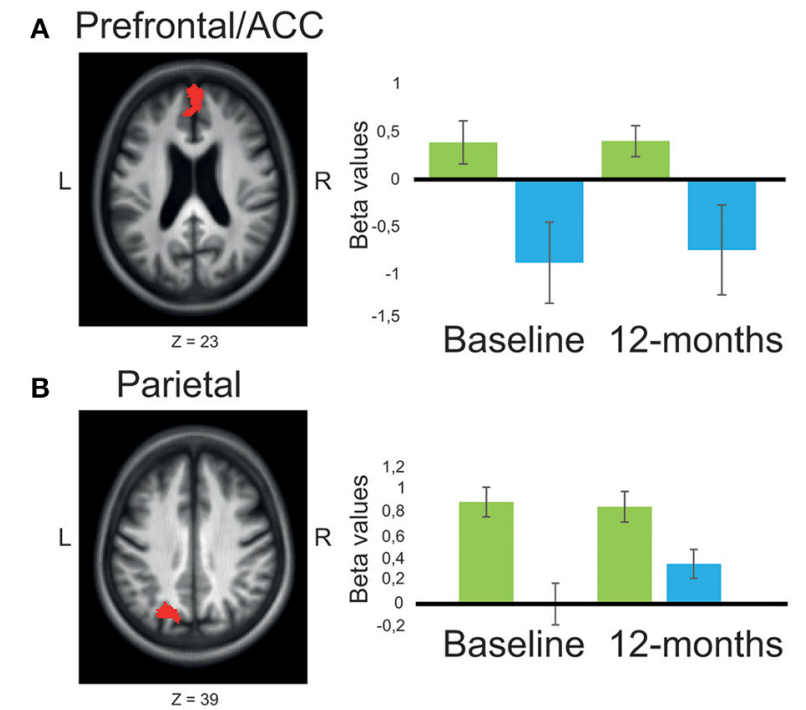

c
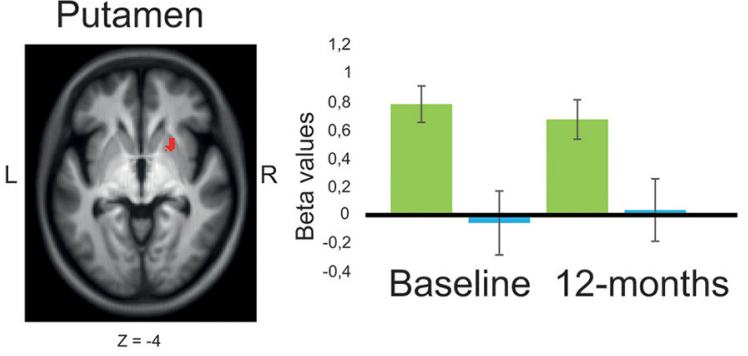

D
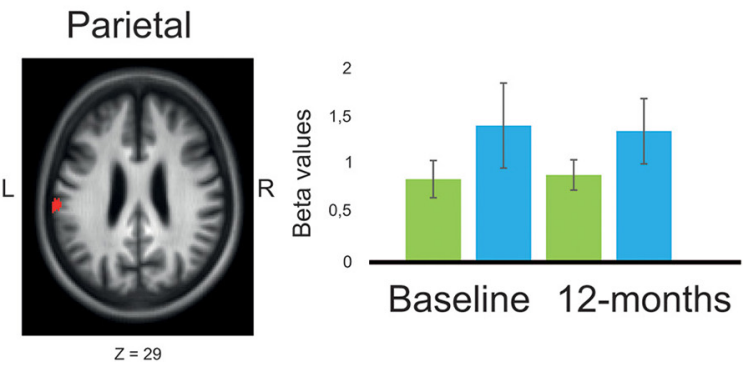

FIGURE 3 | Main effects of group across time-points, comparing BOLD-signal intensity in patients with Parkinson's disease with or without $\mathbf{M C l}$. (A) The presented results are chosen to illustrate fronto-striatal respective posterior cortical circuitry (with two opposite patterns). significant main effect of group (red) was shown in bilateral medial PFC/ACC (A), left superior parietal/occipital cortex (B), and putamen (C), where patients with $\mathrm{MCl}(n=11)$ showed reduced BOLD-signal intensity compared to patients without $\mathrm{MCl}(n=28)$. An opposite pattern was shown in left supramarginal gyrus (D) were patients with $\mathrm{MCl}$ showed larger BOLD-signal intensity compared to patients without $\mathrm{MCl}$. Mean beta values are presented as plots contrasting working-memory 2-back task with the baseline resting condition for patients with Parkinson's disease without $\mathrm{MCl}$ (green), and patients with $\mathrm{MCl}$ (blue). Error bars are $1 \mathrm{SE} . \mathrm{Z}$, anatomical location in Montreal Neurological Institute transversal space. The statistical threshold in the pictures (red clusters) is set to $p<0.005$ to increase visibility.

$\mathrm{PDMCI}^{+}$between right fusiform gyrus and bilateral caudate nucleus (15 voxels located in MNI-space $x=-16, y=18, z=$ 4 , and 13 voxels in $x=18, y=10, z=14)$, and as a trend in right pre-central gyrus (32 voxels in $x=56, y=8, z=40$ ), Less conclusive evidence due to potential influences of the scanner factor, was observed in left pre-central gyrus, and in left superior intraparietal sulcus (Supplementary Note 2). The functional connectivity between right fusiform gyrus and other brain regions did not decline as function of time.

\section{ROI analysis}

In a previous study (Ekman et al., 2012), we reported that $\mathrm{PDMCI}^{+}$under-recruited parts of the ACC and also the right caudate already at the time for initial PD-diagnosis compared to $\mathrm{PDMCI}^{-}$. In keeping with those findings, the group differences remained in parts of the ACC and contiguous areas as shown in the whole-brain analyses of our current study. However, the whole-brain analysis did not reveal any group difference in the caudate, and we therefore applied the right caudate ROI from our previous cross-sectional study (Ekman et al., 2012). The group difference in the right caudate ROI was weakened at follow-up and the main effect of group was now only approaching significance $(p=0.07)$.

\section{DISCUSSION}

In a population-based cohort of newly diagnosed patients with incident $\mathrm{PD}$, we showed that $\mathrm{PDMCI}^{+}$performed inferior across time-points on the fMRI working-memory task compared to $\mathrm{PDMCI}^{-}$. Evaluations of longitudinal changes in functional brain responses showed a group-by-time interaction effect in the right fusiform gyrus, where $\mathrm{PDMCI}^{+}$longitudinally decreased their BOLD-signal intensity whereas $\mathrm{PDMCI}^{-}$were stable across time-points. In addition to the longitudinal change, compared to $\mathrm{PDMCI}^{-}, \mathrm{PDMCI}^{+}$displayed under-recruited BOLD-signal intensity across time in medial prefrontal cortex.

The fusiform gyrus is primarily associated with higher-order visual processing, but also with visual working-memory processing (Cuortney et al., 1997; Wager and Smith, 2003; Chang et al., 2007; Rottschy et al., 2012). It has been hypothesized that working-memory maintenance may involve the same cortical regions that initially process the representations to be held in working-memory (D'Esposito, 2007). We confirmed with the PPI analysis that the right fusiform gyrus is functionally connected with bilateral caudate nucleus, bilateral pre-central gyrus and left intraparietal sulcus during working-memory processing (Gazzaley et al., 2004). Critically, such connectivity was more robust for $\mathrm{PDMCI}^{-}$compared to $\mathrm{PDMCI}^{+}$. The reported alterations in the right fusiform gyrus may thus reflect a longitudinal change in $\mathrm{PDMCI}^{+}$that is related to working-memory maintenance. However, the right fusiform gyrus change might precede additional cognitive decline for $\mathrm{PDMCI}^{+}$at follow-up (Bateman et al., 2012). Structural changes in the fusiform gyrus have been reported for patients with $\mathrm{PD}$ and $\mathrm{MCI}$ compared to patients without MCI (Pagonabarraga et al., 2013), as well as marked reductions in gray matter density in patients with PDD (NaganoSaito et al., 2005; Ramírez-Ruiz et al., 2005). Functional changes have been related to evolving glucose metabolism decline within cognitive networks with both posterior and prefrontal cortical basis in patients with PD (Huang et al., 2007), but impairments in cognitive measures mediated by pathology in posterior cortical regions rather than fronto-striatal regions have been proposed 


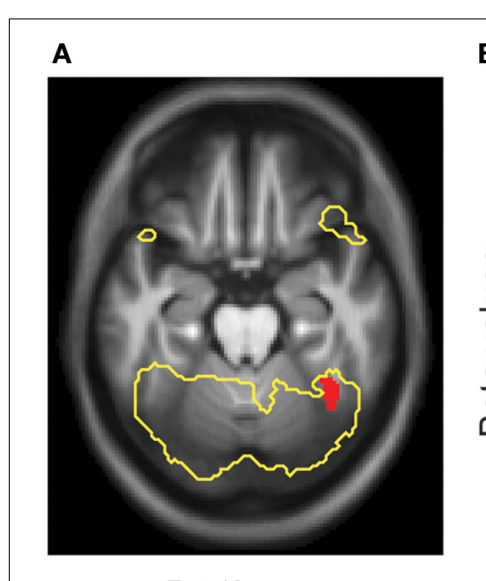

$Z=-19$
B

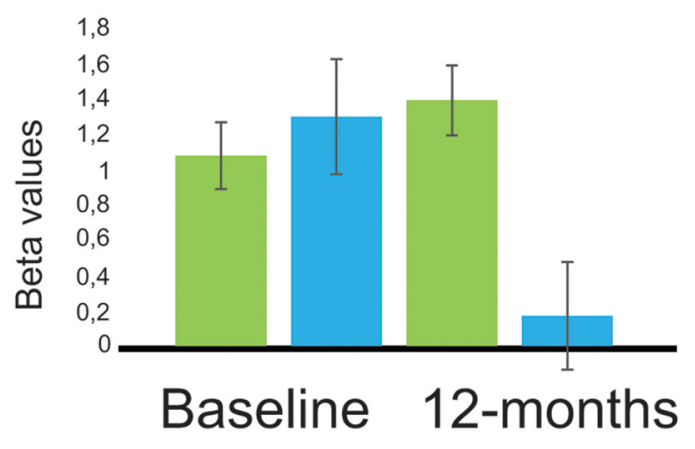

C

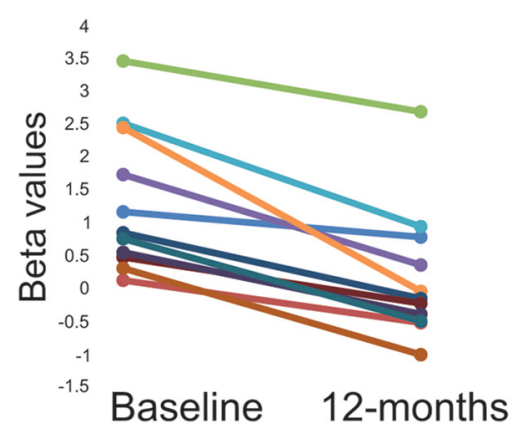

FIGURE 4 | Group-by-time interaction effect, comparing BOLD-signal intensity in patients with Parkinson's disease with or without MCI. (A) A significant group-by-time interaction effect (red) was shown in the right fusiform gyrus comparing patients with Parkinson's disease and $\mathrm{MCl}(n=11)$ to patients without $\mathrm{MCl}(n=28)$. The task-specific activation for all included participants with Parkinson's disease are presented as a yellow outline to illustrate the contrast between 2-back working-memory processing and resting baseline-condition ( $p<0.05$, family-wise error corrected). (B) Mean beta values are presented as plots for patients with Parkinson's disease without $\mathrm{MCl}$ (green), and with $\mathrm{MCl}$ (blue) at both time-points. (C) All patients with Parkinson's disease and $\mathrm{MCl}$ showed a negative slope across time with decreased BOLD-signal intensity (each participants is represented as a line in the figure). Error bars are $1 \mathrm{SE}$. Z, anatomical location in Montreal Neurological Institute transversal space.
Table 3 | Group-by-time interaction effects in BOLD-signal intensity between patients with Parkinson's disease with and without $\mathrm{MCl}$.

\begin{tabular}{|c|c|c|c|c|}
\hline Brain regions & Side & Peak $(x, y, z)$ & $F$ & $K$ \\
\hline \multicolumn{5}{|c|}{$\begin{array}{l}\text { PATIENTS WITH MCI SHOW DECREASED BOLD-SIGNAL INTENSITY } \\
\text { LONGITUDINALLY }\end{array}$} \\
\hline Fusiform gyrus & $\mathrm{R}$ & $38,-48,-22$ & 26.45 & 86 \\
\hline Vermis & $\mathrm{R}$ & $4,-52,-20$ & 19.87 & 19 \\
\hline Inferior temporal* & $\mathrm{L}$ & $-48,-2,-38$ & 13.25 & 23 \\
\hline
\end{tabular}

PATIENTS WITH MCI SHOW INCREASED BOLD-SIGNAL INTENSITY LONGITUDINALLY

$\begin{array}{llccc}\text { Dorsolateral prefrontal* }^{*} & \mathrm{~L} & -42,44,26 & 14.43 & 45 \\ \text { Superior parietal* } & \mathrm{R} & 22,-52,60 & 13.36 & 26\end{array}$

The coordinates $x, y, z$ refers to the anatomical location of the Montreal Neurological Institute space for the clusters local maxima. $p<0.001 ;{ }^{*} p<0.005$. $L$, left; $R$, right; $F$, F-values; $k$, number of voxels.

to predict prodromal PDD (Williams-Gray et al., 2007, 2013). Correspondences of brain atrophy in temporal-parietal regions between patients with PD and Alzheimer's disease have also been reported to predict long-term cognitive decline (Weintraub et al., 2012).

In a previous cross-sectional study, we reported that newly diagnoses drug-naïve patients with $\mathrm{PDMCI}^{+}$under-recruited bilateral ACC and right caudate during working-memory updating (Ekman et al., 2012). In agreement with that, we show in the present study that $\mathrm{PDMCI}^{+}$under-recruited bilateral medial prefrontal cortex (including ACC) compared to $\mathrm{PDMCI}^{-}$, and that the alterations persist across time. ACC is related to high-level cognitive processing (Duncan and Owen, 2000), and PD-related alterations are associated with striatal dopaminergic depletions (Ito et al., 2002), decreased metabolism (Polito et al., 2012), and loss of neuronal integrity (Lewis et al., 2012).

In agreement with our previous cross-sectional study, persistent striatal alterations were shown in the present study, where $\mathrm{PDMCI}^{+}$showed inferior BOLD-signal intensity in right putamen and in the right caudate ROI compared to $\mathrm{PDMCI}^{-}$. However, a main effect of scanner overlapped with the main effect of group in the right putamen, which make this evidence less conclusive, even though large scanner-specific effect sizes were evident. Striatal alterations are repeatedly reported in the early phase of PD in relation to cognitive impairment and depleted dopaminergic circuitry (Sawamoto et al., 2008; Kehagia et al., 2010; Ekman et al., 2012), and dopamine synthesis capacity in the putamen and caudate positively correlates with working-memory capacity (Cools et al., 2008). A weakened group difference was revealed in the right caudate ROI when comparing $\mathrm{PDMCI}^{+}$to $\mathrm{PDMCI}^{-}$longitudinally, potentially reflecting a beneficial medication effect due to onset of anti-parkinsonian treatment. A positive treatment effect might also partly explain the lack of further behavioral decline for $\mathrm{PDMCI}^{+}$on the scanner workingmemory task, and on the cognitive tests outside the scanner. However, anti-parkinsonian dopaminergic medication has previously shown both positive and negative outcomes on cognitive functions (Cools, 2006), and given the relatively small sample size, potential cognitive effects of dopaminergic medication on brain activation is speculative.

The reported alterations might be associated to additional neurochemical dysfunction. Hypo-metabolism have been reported in patients with $\mathrm{PD}$ and multi-domain MCI compared to patients with normal cognition in lateral frontal cortex, ACC, and parietal-temporal-occipital cortices (Lyoo et al., 2010). Patients with PD commonly demonstrate cortical cholinergic dysfunction that is more pronounced in PDD (Bohnen et al., 
2003), and covariance between striatal ${ }^{18} \mathrm{~F}$-fluorodopa (FDOPA) reduction and decreased cortical cholinergic binding in frontal and temporal-parietal cortices have been reported in PDD (Hilker et al., 2005). Furthermore, degeneration of locus coeruleus (LC) might affect executive processes due to deficits in norepinephrine (NE) transmission (Marsh et al., 2009; Vazey and Aston-Jones, 2012). The LC region mainly projects to parietal, temporal, and frontal regions. Thus, the current findings suggest an early evolving pattern in $\mathrm{PDMCI}^{+}$consisting of posterior cortical change that might relate to evolving norepinephrine and/or cholinergic dysfunction.

In contrast to inferior BOLD-signal intensity for $\mathrm{PDMCI}^{+}$, we report group-by-time interaction effects in right parietal cortex indicating up-regulated BOLD-signal intensity between baseline and follow-up for $\mathrm{PDMCI}^{+}$, whereas $\mathrm{PDMCI}^{-}$were stable across time. Additionally, $\mathrm{PDMCI}^{+}$recruited parts of the left supramarginal gyrus to a larger extent than $\mathrm{PDMCI}^{-}$ across time. The supramarginal gyrus has been proposed as part of the phonological loop and is associated to workingmemory span and interference control (Burgess et al., 2011). The reported up-regulations could potentially reflect increased effort in performing the fMRI working-memory task due to evolving cognitive impairments related to fronto-striatal and temporal circuitry. The increased activation might also relate to compensatory regulations for the observed fronto-striatal alterations, and might in part explain the lack of additional behavioral decline at follow-up.

The longitudinal method is a strength as it increases the ability to generalize the reported effects (Raz, 2005; Nyberg et al., 2010), whereas the repeated neuropsychological assessments increases the prognostic accuracy of MCI with respect to prodromal PDD (Pedersen et al., 2013). Data acquisition was conducted on two different scanners and is a limitation that might have induced additional variability. The detection ability is also more restricted in subcortical regions for the $1.5 \mathrm{~T}$ scanner compared to the $3 \mathrm{~T}$ scanner (Nyberg et al., 2007). More importantly, the scanner confound risk making interpretations of group differences unreliable. Critically, the reported differences showed medium to large effect sizes on scanner-separated sub-analyses. Nevertheless, regions showing overlap between group differences and effects of scanner should be interpreted with caution. The relatively small $\mathrm{PDMCI}^{+}$sample is a limitation, and affected the ability to generalize the findings to the general PD-population. Furthermore, our criteria for assessing MCI was not completely fulfilled (i.e., not two measures in each domain) which might have affected detection ability and also prevented sub-analyses of domainspecific MCI.

In conclusion, we report longitudinal BOLD-signal reductions in the right fusiform gyrus for $\mathrm{PDMCI}^{+}$compared to $\mathrm{PDMCI}^{-}$. In addition, compared to $\mathrm{PDMCI}^{-}, \mathrm{PDMCI}^{+}$showed persistent but non-changing under-recruitment across time in fronto-striatal circuitry in relation to deficits in working-memory updating. Taken together, the results are in keeping with the notion that the longitudinal development of cognitive impairment in PD reflect posterior cortical change rather than further fronto-striatal changes. Our population-based longitudinal approach thus adds further knowledge of how task-evoked brain responses relate to cognitive impairments in $\mathrm{PD}$.

\section{DISCLOSURE}

Urban Ekman reports no disclosures. Johan Eriksson reports no disclosures. Lars Forsgren has received honoraria from Orion Pharma and GSK in the past 2 years. Magdalena E. Domellöf reports no disclosures. Eva Elgh reports no disclosures. Anders Lundquist reports no disclosures. Lars Nyberg reports no disclosures.

\section{ACKNOWLEDGMENTS}

The authors would like to thank Micael Andersson, Alireza Salami, Jan Linder, the staff at Umeå Center for Functional Brain Imaging, and the collaborators in the NYPUM project. This work was funded by grants from the Swedish Medical Research Council, Swedish Parkinson Foundation, Swedish Parkinson's disease Association, Umeå University, Kempe Foundation, Lions Clubs Sweden's Foundation for Research in Age-Related Diseases, Foundation for Clinical Neuroscience at Umeå University Hospital, Västerbotten County Council (ALF), King Gustaf V's and Queen Victoria's Freemason Foundation, Knut and Alice Wallenberg Foundation, and Swedish Brain Power.

\section{SUPPLEMENTARY MATERIAL}

The Supplementary Material for this article can be found online at: http://www.frontiersin.org/journal/10.3389/fnins.2014. 00207/abstract

\section{REFERENCES}

Aarsland, D., Bronnick, K., Williams-Gray, C., Weintraub, D., Marder, K., Kulisevsky, J., et al. (2010). Mild cognitive impairment in Parkinson's disease: a multicenter pooled analysis. Neurology 75, 1062-1069. doi: 10.1212/WNL.0b $013 \mathrm{e} 3181 \mathrm{f} 39 \mathrm{~d} 0 \mathrm{e}$

Abdulkadir, A., Mortamet, B., Vemuri, P., Clifford, R. J., Krueger, G., and Klöppel, S. (2011). Effects of hardware heterogeneity on the performance of SVM Alzheimer's disease classifier. NeuroImage 58, 785-792. doi: 10.1016/j.neuroimage.2011.06.029

Bateman, R. J., Xiong, C., Benzinger, T. L., Fagan, A. M., Goate, A., Fox, N. C., et al. (2012). Clinical and biomarker changes in dominantly inherited Alzheimer's disease. N. Engl. J. Med. 367, 795-804. doi: 10.1056/NEJMoa1202753

Bohnen, N. I., Kaufer, D. I., Ivanco, L. S., Lopresti, B., Koeppe, R. A., Davis, J. G., et al. (2003). Cortical cholinergic function is more severely affected in parkinsonian dementia than in Alzheimer disease: an in vivo positron emission tomographic study. Arch. Neurol. 60, 1745-1748. doi: 10.1001/archneur.60.12.1745

Broeders, M., de Bie, R. M. A., Belseboer, D. C., Speelman, J. D., Muslimovic, D., and Schmand, B. (2013). Evolution of mild cognitive impairment in Parkinson's disease. Neurology 81, 346-352. doi: 10.1212/WNL.0b013e31829c5c86

Burgess, G. C., Gray, J. R., Conway, A. R. A., and Braver, T. S. (2011). Neural mechanisms of interference control underlie the relationship between fluid intelligence and working memory span. J. Exp. Psychol. 140, 674-692. doi: 10.1037/a0024695

Chang, C., Crottaz-Herbette, S., and Menon, V. (2007). Temporal dynamics of basal ganglia response and connectivity during verbal working memory. Neuroimage 34, 1253-1269. doi: 10.1016/j.neuroimage.2006.08.056

Cools, R. (2006). Dopaminergic modulation of cognitive function - implications for L-DOPA treatment in Parkinson's disease. Neurosci. Biobehav. Rev. 30, 1-23. doi: 10.1016/j.neubiorev.2005.03.024

Cools, R., Gibbs, S. E., Miyakawa, A., Jagust, W., and D'esposito, M. (2008). Working memory capacity predicts dopamine synthesis capacity in the human striatum. J. Neurosci. 28, 1208-1212. doi: 10.1523/JNEUROSCI.4475-07.2008

Cuortney, S. M., Ungerleider, L. G., Keil, K., and Haxby, J. V. (1997). Transient and sustained activity in a distributed neural system for human working memory. Nature 386, 608-611. doi: 10.1038/386608a0

D'Esposito, M. (2007). From cognitive to neural models of working memory. Philos. Trans. R. Soc. Lond. B Biol. Sci. 362, 761-772. doi: 10.1098/rstb.2007.2086

Duncan, J., and Owen, A. M. (2000). Common regions of the human frontal lobe recruited by diverse cognitive demands. Trends Neurosci. 23, 475-483. doi: 10.1016/S0166-2236(00)01633-7 
Ekman, U., Eriksson, J., Forsgren, L., Jakobson, S., Riklund, K., and Nyberg, L. (2012). Functional brain activity and presynaptic dopamine uptake in patients with Parkinson's disease and mild cognitive impairment: a cross-sectional study. Lancet Neurol. 11, 679-687. doi: 10.1016/S1474-4422(12)70138-2

Emre, M., Aarsland, D., Brown, R., Burn, D. J., Duyckaerts, C., Mizuno, Y., et al. (2007). Clinical diagnostic criteria for dementia associated with Parkinson's disease. Mov. Disord. 22, 1689-1707. doi: 10.1002/mds.21507

Friston, K. J., Buechel, C., Fink, G. R., Morris, J., Rolls, E., and Dolan, R. J. (1997). Psychophysiological and modulatory interactions in neuroimaging. Neuroimage 6, 218-229. doi: 10.1006/nimg.1997.0291

Friston, K. J., Holmes, A. P., Worsley, K. J., Poline, J. P., Frith, C. D., and Frackowiak, R. S. J. (1995). Statistical parametric maps in functional imaging: a general linear approach. Hum. Brain Mapp. 2, 189-210. doi: 10.1002/hbm.460020402

Gazzaley, A., Rissman, J., and D'Esposito, M. (2004). Functional connectivity during working memory maintenance. Cogn. Affect. Behav. Neurosci. 4, 580-599. doi: 10.3758/CABN.4.4.580

Gibb, W. R., and Lees, A. J. (1988). The relevance of the Lewy body to the pathogenesis of idiopathic Parkinson's disease. J. Neurol. Neurosurg. Psychiatry 51, 745-752. doi: 10.1136/jnnp.51.6.745

Hilker, R., Thomas, A. V., Klein, J. C., Weisenbach, S., Kalbe, E., Burghaus, L., et al. (2005). Dementia in Parkinson's disease: functional imaging of cholinergic and dopaminergic pathways. Neurology 65, 1716-1722. doi: 10.1212/01.wnl.0000191154.78131.f6

Huang, C., Tang, C., Felgin, A., Lesser, M., Ma, Y., Pourfar, M., et al. (2007). Changes in network activity with the progression of Parkinson's disease. Brain 130, 1834-1846. doi: 10.1093/brain/awm086

Ito, K., Nagano-Saito, A., Kato, T., Arahata, Y., Nakamura, A., and Kawasumi, Y. (2002). Striatal and extrastriatal dysfunction in Parkinson's disease with dementia: a $6-\left[{ }^{18} \mathrm{~F}\right]$ fluoro-L-dopa PET study. Brain 125, 1358-1365. doi: 10.1093/brain/awf134

Janvin, C. C., Larsen, J. P., Aarsland, D., and Hugdahl, K. (2006). Subtypes of mild cognitive impairment in Parkinson's disease: progression to dementia. Mov. Disord. 21, 1343-1349. doi: 10.1002/mds.20974

Kehagia, A. A., Barker, R. A., and Robbins, T. W. (2010). Neuropsychological and clinical heterogeneity of cognitive impairment and dementia in patients with Parkinson's disease. Lancet Neurol. 9, 1200-1213. doi: 10.1016/S14744422(10)70212-X

Lewis, S. J. G., Dove, A., Robbins, T. W., Barker, R. A., and Owen, A. M. (2003). Cognitive impairments in early Parkinson's disease are accompanied by reductions in activity in frontostriatal neural circuitry. J. Neurosci. 23, 6351-6356.

Lewis, S. J. G., Shine, J. M., Duffy, S., Halliday, G., and Naismith, S. L. (2012). Anterior cingulate integrity: executive and neuropsychiatric features in Parkinson's disease. Mov. Disord. 27, 1262-1267. doi: 10.1002/mds. 25104

Litvan, I., Goldman, J. G., Tröster, A. I., Schmand, B. A., Weintraub, D., Petersen, R. C., et al. (2012). Diagnostic criteria for mild cognitive impairment in Parkinson's disease: movement disorder society task force guidelines. Mov. Disord. 27, 349-356. doi: 10.1002/mds.24893

Lyoo, C. H., Jeong, Y., Ryu, Y. H., Rinne, J. O., and Lee, M. S. (2010). Cerebral glucose metabolism of Parkinson's disease patients with mild cognitive impairment. Eur. Neurol. 64, 65-73. doi: 10.1159/000315036

Marchewka, A., Kherif, F., Krueger, G., Grabowska, A., Frackowiak, R., and Draganski, B. (2014). Influence of magnetic field strength and image registration strategy on voxel-based morphometry in a study of Alzheimer's disease. Hum. Brain Mapp. 35, 1865-1874. doi: 10.1002/hbm. 22297

Marklund, P., Larsson, A., Elgh, E., Linder, J., Riklund, K. A., Forsgren, L., et al. (2009). Temporal dynamics of basal ganglia under-recruitment in Parkinson's disease: transient caudate abnormalities during updating of working memory. Brain 132, 336-346. doi: 10.1093/brain/awn309

Marsh, L., Biglan, K., Gerstenhaber, M., and Williams, J. R. (2009). Atomoxetine for the treatment of executive dysfunction in Parkinson's disease: a pilot open-label study. Mov. Disord. 24, 277-282. doi: 10.1002/mds.22307

Mattay, V. S., Tessitore, A., and Callicott, J. H. (2002). Dopaminergic modulation of cortical function in patients with Parkinson's disease. Ann. Neurol. 51, 156-164. doi: 10.1002/ana.10078

Monchi, O., Petrides, M., Mejia-Constain, B., and Strafella, A. P. (2007). Cortical activity in Parkinson's disease during executive processing depends on striatal involvement. Brain 130, 233-244. doi: 10.1093/brain/awl326
Monchi, O., and Stoessl, A. J. (2012). Imaging neural correlates of mild cognitive impairment in Parkinson's disease. Lancet Neurol. 11, 653-655. doi: 10.1016/S1474-4422(12)70162-X

Montgomery, S. A., and Åsberg, M. (1979). A new depression scale designed to be sensitive to change. Br. J. Psychiatry 134, 382-389. doi: 10.1192/bjp.134.4.382

Nagano-Saito, A., Habak, C., Mejía-Constaín, B., Degroot, C., Monetta, L., Jubault, T., et al. (2014). Effect of mild cognitive impairment on the patterns of neural activity in early Parkinson's disease. Neurobiol. Aging 35, 223-231. doi: 10.1016/j.neurobiolaging.2013.06.025

Nagano-Saito, A., Washimi, Y., Arahata, Y., Kachi, T., Lerch, J. P., Evans, A. C., et al. (2005). Cerebral atrophy and its relation to cognitive impairment in Parkinson disease. Neurology 64, 224-229. doi: 10.1212/01.WNL.0000149510.41793.50

Nyberg, L., Larsson, A., Eriksson, J., Birgander, R., Sundström, T., and Riklund, K. (2007). "Comparing 1.5T and 3T bold fMRI imaging of finger tapping with familiar and novel sequences," in Neuroscience Imaging Research Trends, eds B. Schaller (New York, NY: Nova Science Publishers), 161-172.

Nyberg, L., Salami, A., Andersson, M., Eriksson, J., Kalpouzos, G., Kauppi, K., et al. (2010). Longitudinal evidence for diminished frontal cortex function in aging. Proc. Natl. Acad. Sci. U.S.A. 107, 22682-22686. doi: 10.1073/pnas.1012651108

Owen, A. M. (2004). Cognitive dysfunction in Parkinson's disease: the role of frontostriatal circuitry. Neuroscientist 10, 525-537. doi: 10.1177/1073858404266776

Pagonabarraga, J., Corcuera-Solano, I., Vives-Gilabert, Y., Llebaria, G., GarciaSanchez, C., and Pascual-Sedano, B. (2013). Pattern of regional cortical thinning associated with cognitive deterioration in Parkinson's disease. PLOS ONE 8:e54980. doi: 10.1371/journal.pone.0054980

Pedersen, K. F., Larsen, J. P., Tysnes, O.-B., and Alves, G. (2013). Prognosis of mild cognitive impairment in early Parkinson's disease: the Norwegian Park West Study. JAMA Neurol. 70, 580-586. doi: 10.1001/jamaneurol.2013.2110

Polito, C., Berti, V., Ramat, S., Vanzi, E., De Cristofaro, M. T., Pellicanò, G., et al. (2012). Interaction of caudate dopamine depletion and brain metabolic changes with cognitive dysfunction in early Parkinson's disease. Neurobiol. Aging 33, 206.e29-206.e39. doi: 10.1016/j.neurobiolaging.2010.09.004

Ramírez-Ruiz, B., Martí, M. J., Tolosa, E., Bartrés-Faz, D., Summerfield, C., Salgado-Pineda, P., et al. (2005). Longitudinal evaluation of cerebral morphological changes in Parkinson's disease with and without dementia. J. Neurol. 252, 1345-1352. doi: 10.1007/s00415-005-0864-2

Raz, N. (2005). Regional brain changes in aging healthy adults: general trends, individual differences and modifiers. Cereb. Cortex 15, 1676-1689. doi: 10.1093/cercor/bhi044

Richard, E., and Brayne, C. (2014). Mild cognitive impairment - not always what it seems. Nat. Rev. Neurol. 10, 130-131. doi: 10.1038/nrneurol.2014.23

Rinne, J. O., Portin, R., Ruottinen, H., Nurmi, E., Bergman, J., Haaparanta, M., et al. (2000). Cognitive impairment and the brain dopaminergic system in Parkinson's disease. Arch. Neurol. 57, 470-475. doi: 10.1001/archneur.57.4.470

Rottschy, C., Langner, R., Dogan, I., Reetz, K., Laird, A. R., Schultz, J. B., et al. (2012). Modelling neural correlates of working memory: a coordinate-based meta-analysis. Neuroimage 60, 830-846. doi: 10.1016/j.neuroimage.2011.11.050

Sawamoto, N., Piccini, P., Hotton, G., Pavese, N., Thielemans, K., and Brooks, D. J. (2008). Cognitive deficits and striato-frontal dopamine release in Parkinson's disease. Brain 131, 1294-1302. doi: 10.1093/brain/awn054

Stonnington, C. M., Tan, G., Klöppel, S., Chu, C., Draganski, B., Jack, C. R. Jr, et al. (2008). Interpreting scan data acquired from multiple scanners: a study with Alzheimer's disease. Neuroimage 39, 1180-1185. doi: 10.1016/j.neuroimage.2007.09.066

Svenningsson, P., Westman, E., Ballard, C., and Aarsland, D. (2012). Cognitive impairment in patients with Parkinson's disease: diagnosis, biomarkers, and treatment. Lancet Neurol. 11, 697-707. doi: 10.1016/S1474-4422(12) 70152-7

Tomlinson, C. L., Stowe, R., Patel, S., Rick, C., Gray, R., and Clarke, C. E. (2010). Systematic review of levodopa dose equivalency reporting in Parkinson's disease. Mov. Disord. 25, 2649-2653. doi: 10.1002/mds.23429

Vazey, E. M., and Aston-Jones, G. (2012). The emerging role of norepinephrine in cognitive dysfunctions of Parkinson's disease. Front. Behav. Neurosci. 6:48. doi: 10.3389/fnbeh.2012.00048

Wager, T. D., and Smith, E. E. (2003). Neuroimaging studies of working memory: a meta-analysis. Cogn. Affect. Behav. Neurosci. 3, 255-274. doi: 10.3758/CABN.3.4.255

Weintraub, D., Dietz, N., Duda, J. E., Wolk, D. A., Doshi, J., Xie, S. X., et al. (2012). Alzheimer's disease pattern of brain atrophy predicts cognitive 
decline in Parkinson's disease. Brain 135, 170-180. doi: 10.1093/brain/ awr277

Williams-Gray, C. H., Foltynie, T., Brayne, C. E., Robbins, T. W., and Barker, R. A. (2007). Evolution of cognitive dysfunction in an incident Parkinson's disease cohort. Brain 130, 1787-1798. doi: 10.1093/brain/ awm111

Williams-Gray, C. H., Mason, S. L., Evans, J. R., Foltynie, T., Brayne, C., Robbins, T. W., et al. (2013). The CamPaIGN study of Parkinson's disease: 10-year outlook in an incident population-based cohort. J. Neurol. Neurosurg. Psychiatry 84, 1258-1264. doi: 10.1136/jnnp-2013-305277

Conflict of Interest Statement: The authors declare that the research was conducted in the absence of any commercial or financial relationships that could be construed as a potential conflict of interest.
Received: 05 May 2014; accepted: 30 June 2014; published online: 29 July 2014.

Citation: Ekman U, Eriksson J, Forsgren L, Domellöf ME, Elgh E, Lundquist A and Nyberg L (2014) Longitudinal changes in task-evoked brain responses in Parkinson's disease patients with and without mild cognitive impairment. Front. Neurosci. 8:207. doi: $10.3389 /$ fnins.2014.00207

This article was submitted to Neurodegeneration, a section of the journal Frontiers in Neuroscience.

Copyright (C) 2014 Ekman, Eriksson, Forsgren, Domellöf, Elgh, Lundquist and Nyberg. This is an open-access article distributed under the terms of the Creative Commons Attribution License (CC BY). The use, distribution or reproduction in other forums is permitted, provided the original author(s) or licensor are credited and that the original publication in this journal is cited, in accordance with accepted academic practice. No use, distribution or reproduction is permitted which does not comply with these terms. 